\title{
EUVE J0825-16.3 and EUVE J1501-43.6: Two dMe Double-Lined Spectroscopic Binaries
}

\author{
D. Montes ${ }^{1}$, I. Crespo-Chacón ${ }^{1}$, M.C. Gálvez ${ }^{1,2}$, \\ and M.J. Fernández-Figueroa ${ }^{1}$ \\ ${ }^{1}$ Departamento de Astrofísica, Facultad de Ciencias Físicas, \\ Universidad Complutense de Madrid, Spain \\ email: dmg@astrax.fis.ucm.es \\ ${ }^{2}$ Astronomy Department, University of Florida, Bryant Space Science Center, \\ Gainesville, Florida, USA
}

\begin{abstract}
High-resolution echelle spectroscopic observations taken with the FEROS spectrograph at the ESO 2.2-m telescope confirm the binary nature of the dMe stars EUVE J0825-16.3 and EUVE J1501-43.6, previously reported by Christian \& Mathioudakis (2002). In these binary systems, emission of similar intensity from both components is detected in the Na I $\mathrm{D}_{1}$ \& $\mathrm{D}_{2}$, He I $\mathrm{D}_{3}$, Ca II H\&K, Ca II IRT and Balmer lines. We have determined precise radial velocities by cross-correlation with radial velocity standard stars, which have allowed us to obtain for the first time the orbital solution of these systems. Both binaries consist of two nearly equal M0V components with an orbital period shorter than 3.5 days. We have analyzed the behaviour of the chromospheric activity indicators (variability and possible flares). In addition, we have determined its rotational velocity and kinematics.
\end{abstract}

Keywords. stars: binaries: spectroscopic, stars: activity, stars: atmospheres, stars: chromospheres, stars: flare, stars: late-type, stars: fundamental parameters

\section{Observations}

Spectroscopic observations (high-resolution echelle spectra) of these stars were obtained during one observing run from 18 to 22 February 2005 using the 2.2-m telescope at the European Southern Observatory, ESO (La Silla, Chile). We have used the FEROS (Fiber-fed Extended Range Optical Spectrograph) linked to the Cassegrain focus of the $2.2 \mathrm{~m}$ telescope, in unique fiber modus, with CCD $(2048 \times 4096,0.15 \mathrm{~mm} / \mathrm{pixel})$. This configuration provides a resolution of 48000 and a spectral range from 3500 to $9000 \AA$, including lines from Ca II H\&K (3933, $3968 \AA)$ to Ca II IRT $(8498,8542,8662 \AA)$, in a total of 39 orders. A total of 5 spectra of EUVE J0825-16.3 and 6 of EUVE J1501-43.6 has been taken during the 5 nights of observations. Reference stars of similar spectral type and radial velocity standard stars have also been observed with the same configuration.

\section{EUVE J0825-16.3}

EUVE J0825-16.3 is an extreme ultraviolet source detected during the all-sky surveys of the Extreme Ultraviolet Explorer (EUVE) coinciding with the position of a soft X-ray source (1RXS J082551.4-162244) detected by ROSAT PSPC (Craig et al. 1997). Using low resolution spectroscopy, Craig et al. (1997) classified this source as a dM0e with the Ca II H\&K and Balmer lines in emission. Later, two high-resolution spectra reported by Christian \& Mathioudakis (2002) identified it as a double-lined spectroscopic 


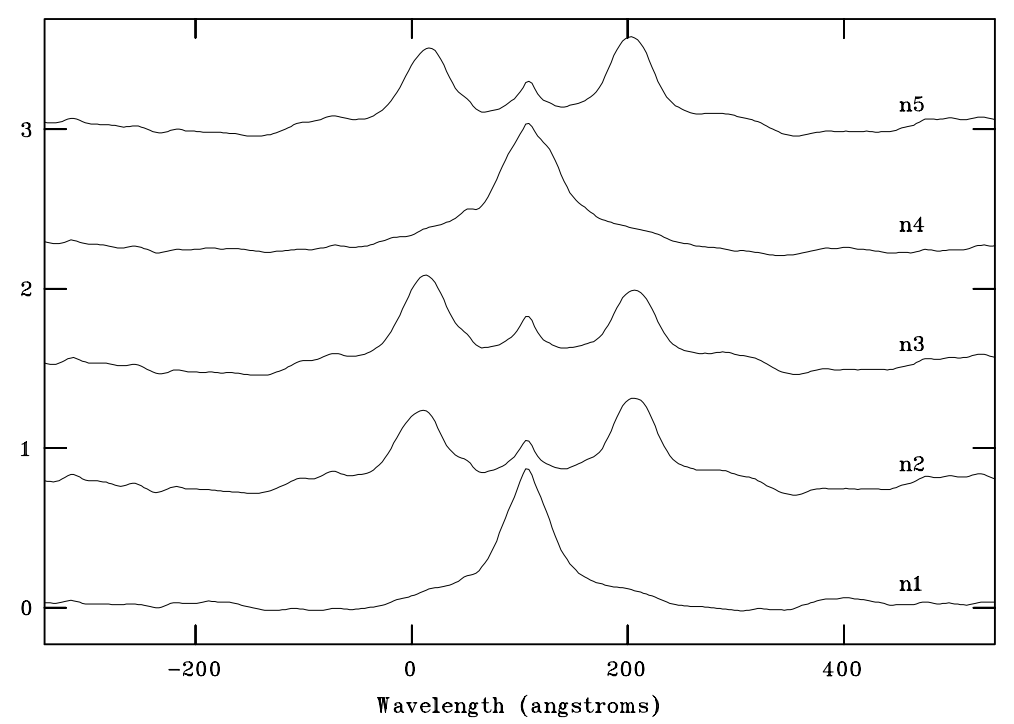

Figure 1. CCF (Cross-Correlation Function) of the spectra of EUVE J0825-16.3 with the spectrum of a radial velocity standard star. Note the three peaks, that indicate that there are three stars contributing to the observed spectrum. The radial velocity derived for the tertiary component does not change during the 5 nights of observations and seems to be equal to the centre of mass radial velocity of the primary and secondary components (see the position of the CCF of primary and secondary components the nights 1 and 4 that are in conjunction).

binary (SB2) with strong Balmer emission from both components, but they did not obtain the orbital solution. Also, these authors determined a rotational velocity $(v \sin i=$ $\left.20 \mathrm{~km} \mathrm{~s}^{-1}\right)$, but they did not detect the lithium line $(6708 \AA)$. Our spectra demonstrate that EUVE J0825-16.3 actually is a triple system. In addition to the double lines reported by Christian \& Mathioudakis (2002) we have identified the signatures of a third object contributing to the observed spectrum (see Figures 1 and 2).

Probably, what we are observing is a visual binary (which is not resolved in the fiber of the spectrograph) where the A component is the short-period SB2 binary (primary and secondary components observed in the spectra) and the B component is the dim tertiary component detected in the spectra with the same radial velocity as the centre of mass of the SB2 system. These two unresolved components could be the two components identified by the Hipparcos satellite (ESA 1997) in the star BD-15 2429 (HIP 41322, NLTT 19515, PPM 715320), which have exactly the same coordinates as our source. Hipparcos reported for these components (CCDM 08259-1623) an angular separation of $0^{\prime \prime} .845$, a position angle of $258^{\circ}$, and a magnitude difference of $2.37 \mathrm{mag}$, which are consistent with our observations.

\subsection{Orbital Solution EUVE J0825-16.3}

Heliocentric radial velocities of the three components of EUVE J0825-16.3 have been determined by using the cross-correlation technique. The spectra were cross-correlated order by order, using the routine FXCOR in IRAF, against spectra of radial velocity standards of similar spectral types. The velocity is derived from the position of the cross-correlation peak (see the CCF in Figure 1). Uncertainties in the derived velocities have been estimated from the width of the cross-correlation peak and the inter-order agreement in the derived velocities.

Using these radial velocities we have determined the orbital solution of the SB2 system 


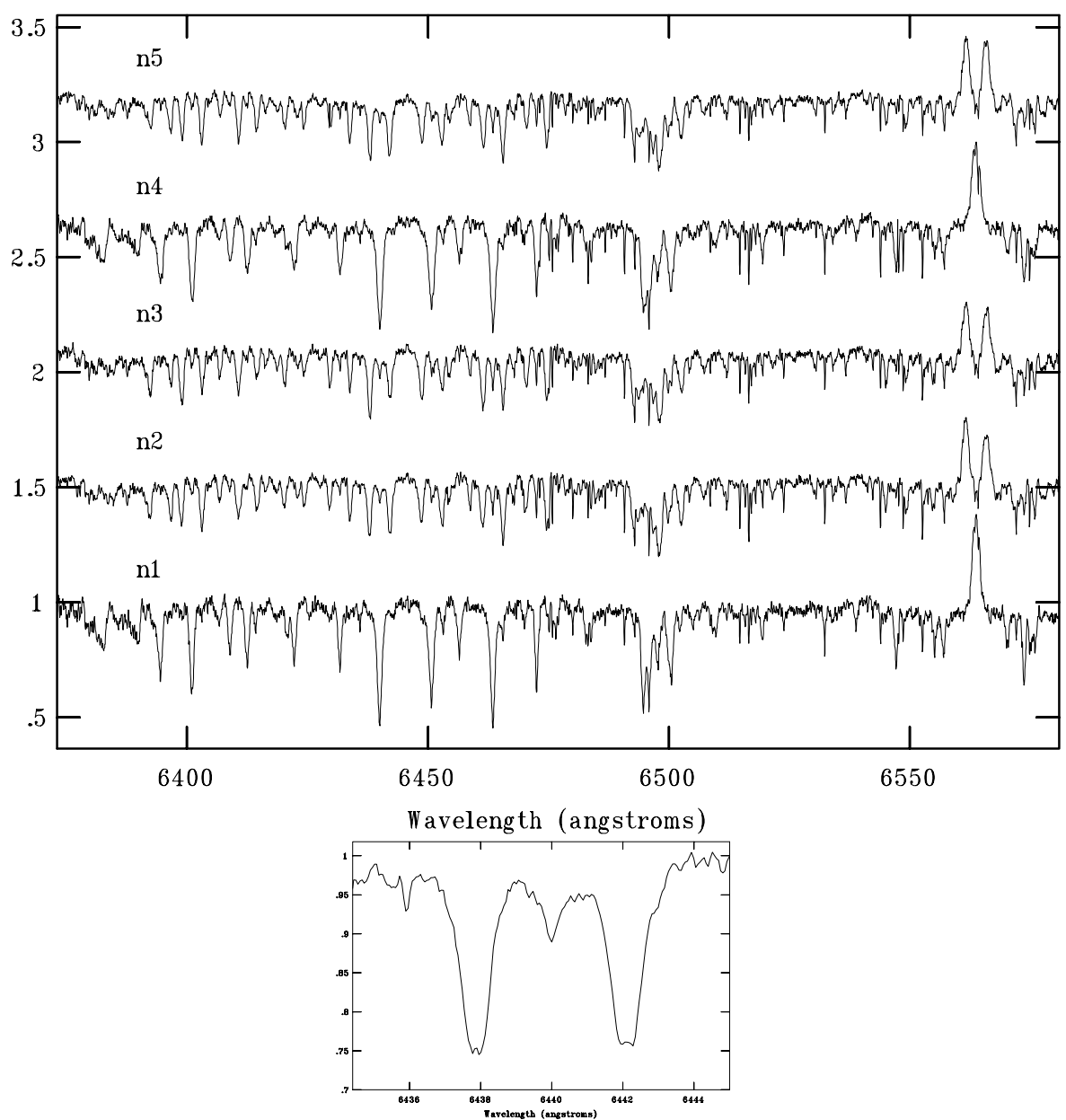

Figure 2. High-resolution spectra of EUVE J0825-16.3 in the $\mathrm{H} \alpha$ line region during our five nights of observations. Note the $\mathrm{H} \alpha$ emission of similar intensity coming from the two main components of the system: primary and secondary. The tertiary component (see Figure 1) is also detected in the photospheric absorption lines (see the zoom of the Ca I $6439 \AA$ line region in the bottom panel).

(see Table 1 and Figure 3). We have obtained a nearly circular orbit with an orbital period of 1.51 days. The resulting minimum masses $M \sin ^{3} i \approx 0.66 M_{\odot}$ and mass ratio $q \approx 1$ are compatible with two nearly equal components of spectral type very similar to the derived from our spectra $(\mathrm{K} 7 \mathrm{~V}-\mathrm{M} 0 \mathrm{~V})$.

\section{EUVE J1501-43.6}

EUVE J1501-43.6 (RE J1501-433) is an extreme ultraviolet and soft X-ray source detected during the all-sky surveys of the Extreme Ultraviolet Explorer (EUVE) and ROSAT PSPC (Lampton et al. 1997). Recently, Christian \& Mathioudakis (2002), using two high-resolution spectra, identified it as a double-lined spectroscopic binary (SB2) with both components of spectral type dM:e, strong Balmer emission lines, rotational velocity $v \sin i<5 \mathrm{~km} \mathrm{~s}^{-1}$, and the lithium line $(6708 \AA)$ with a $\mathrm{EW}<20 \mathrm{~m} \AA$. Although these authors mentioned a possible period of approximately 1 day, they did not obtain 

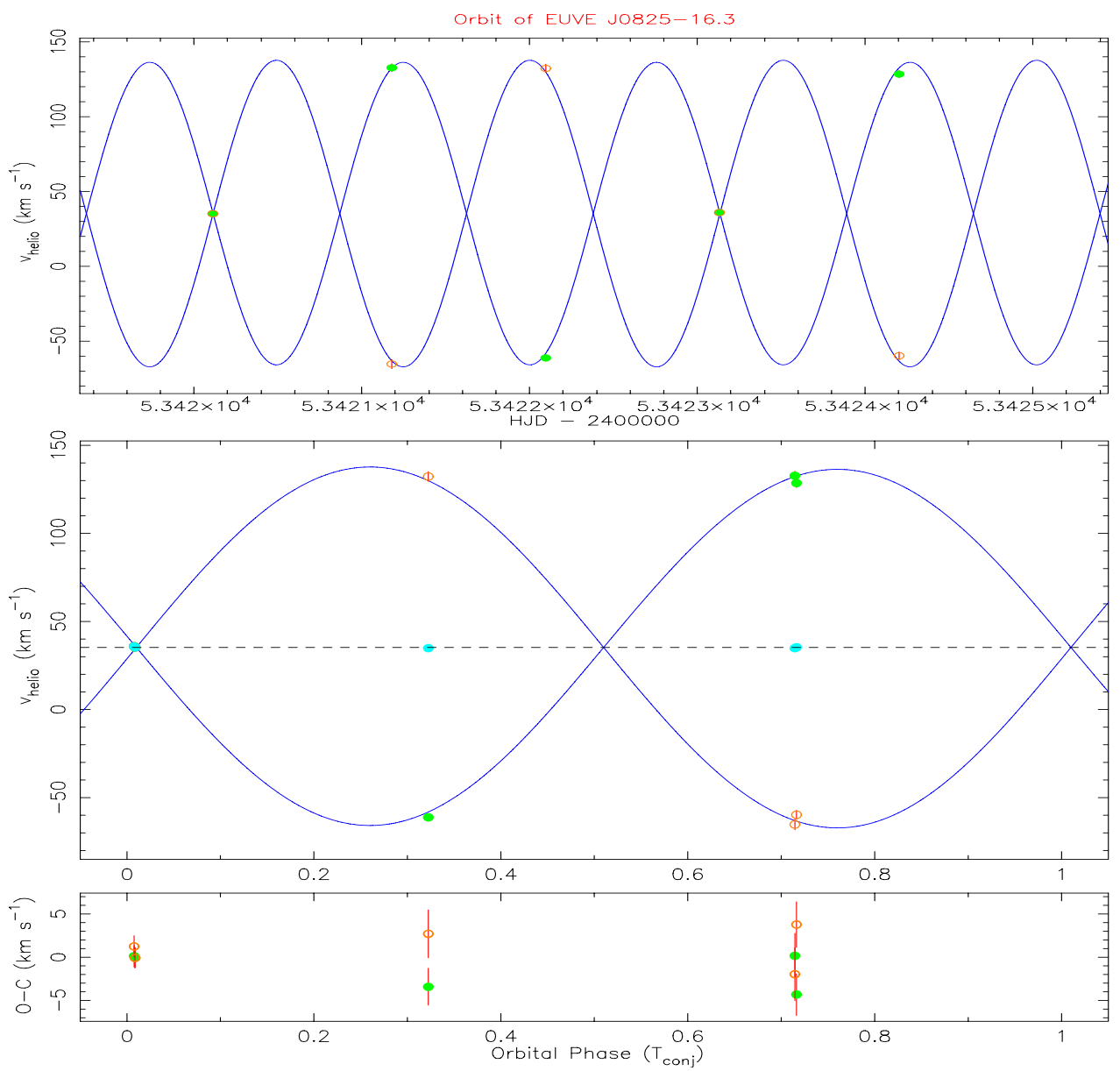

Figure 3. Heliocentric radial velocities determined by us vs. HJD (upper panel) and vs. orbital phase (lower panel). Our observations cover 3 orbital periods. Green solid circles represent the primary (the component with lower chromospheric emission in the SB2 system) and red open circles the secondary. We have also plotted the radial velocity of the tertiary (blue solid circles), as it can be seen, its velocity remains equal to that of the centre of mass of the SB2 system during the 5 days of observations. The blue solid line is the obtained orbital solution.

the orbital solution. Our spectra, taken during 5 nights, confirm the SB2 nature of this system. The near to conjunction spectra obtained at night 1 and 4 (see Figures 4 and 5) suggest a period of approximately 3 days, which is confirmed by the orbital solution reported below. By comparison with several spectral type reference stars, we have obtained that the spectral type of both components is between M2V and M3V. Strong emission from both components is observed in the Ca II H\&K lines, all the Balmer lines (see, for instance, $\mathrm{H} \alpha$ in Figure 4), the Ca II IRT lines, as well as the $\mathrm{Na} \mathrm{I}_{1}, \mathrm{D}_{2}$, and the He I $\mathrm{D}_{3}$ lines (Figure 5). The emission lines in both components have similar intensity, but the secondary component has a slightly large intensity in all the orbital phases. The $\mathrm{H} \alpha$ line of both components exhibits a central self-absorption (see Figure 4).

\subsection{Orbital Solution EUVE J1501-43.6}

Heliocentric radial velocities of the two components of EUVE J1501-43.6 have been determined by using the cross-correlation technique as in the case of EUVE J0825-16.3. 
Table 1. Orbital solution of EUVE J0825-16.3

\begin{tabular}{lccc}
\hline Element & Value & Uncertainty & Units \\
\hline$P$ & 1.5105 & 0.0014 & days \\
$T_{0}$ (peri) & 2453418.5898 & 0.0034 & HJD \\
$e$ & 0.0 & 0.0 & \\
$K_{1}$ & 101.08 & 1.44 & $\mathrm{~km} \mathrm{~s}^{-1}$ \\
$K_{2}$ & 102.42 & 2.69 & $\mathrm{~km} \mathrm{~s}^{-1}$ \\
$\gamma$ & 35.27 & 0.53 & $\mathrm{~km} \mathrm{~s}^{-1}$ \\
$q=M_{1} / M_{2}$ & 1.013 & 0.022 & \\
$a_{1} \sin i$ & 2.100 & 0.030 & $10^{6} \mathrm{~km}$ \\
$a_{2} \sin i$ & 2.127 & 0.056 & $10^{6} \mathrm{~km}$ \\
$a \sin i$ & 4.227 & 0.064 & $10^{6} \mathrm{~km}$ \\
$"$ & 0.0283 & & $\mathrm{AU}$ \\
& 6.0732 & & $\mathrm{R}_{\odot}$ \\
$M_{1} \sin ^{3} i$ & 0.664 & 0.032 & $M_{\odot}$ \\
$M_{2} \sin ^{3} i$ & 0.655 & 0.031 & $M_{\odot}$ \\
$\mathrm{f}(M)$ & 0.1616 & 0.0069 & $M_{\odot}$ \\
\hline
\end{tabular}

Using these radial velocities we have determined the orbital solution of EUVE J1501-43.6 (see Table 2 and Figure 6 ). We have obtained a nearly circular orbit with an orbital period of 3.23 days. The resulting minimum masses $M \sin ^{3} i \approx 0.39 M_{\odot}$ and mass ratio $q \approx 1$ are compatible with two nearly equal M2-M3V components, in agreement with the spectral types determined from the observed spectra.

This binary system is very similar to LU Vel (GJ 375), which also consists of two nearly equal active M3.5V components (see Montes et al. 2006).

Table 2. Orbital solution of EUVE J1501-43.6

\begin{tabular}{lccc}
\hline Element & Value & Uncertainty & Units \\
\hline$P$ & 3.2327 & 0.0046 & days \\
$T_{0}$ (peri) & 2453413.6484 & 0.0087 & $\mathrm{HJD}$ \\
$e$ & 0.0 & 0.0 & \\
$K_{1}$ & 66.56 & 0.40 & $\mathrm{~km} \mathrm{~s}^{-1}$ \\
$K_{2}$ & 66.94 & 0.74 & $\mathrm{~km} \mathrm{~s}^{-1}$ \\
$\gamma$ & -5.82 & 0.20 & $\mathrm{~km} \mathrm{~s}^{-1}$ \\
$q=M_{1} / M_{2}$ & 1.0057 & 0.0093 & \\
$a_{1} \sin i$ & 2.959 & 0.019 & $10^{6} \mathrm{~km}$ \\
$a_{2} \sin i$ & 2.976 & 0.033 & $10^{6} \mathrm{~km}$ \\
$a \sin i$ & 5.935 & 0.038 & $10^{6} \mathrm{~km}$ \\
$"$ & 0.0397 & & $\mathrm{AU}$ \\
& 8.527 & & $\mathrm{R} \odot$ \\
$M_{1} \sin ^{3} i$ & 0.3996 & 0.0082 & $M_{\odot}$ \\
$M_{2} \sin ^{3} i$ & 0.3973 & 0.0081 & $M_{\odot}$ \\
$\mathrm{f}(M)$ & 0.0988 & 0.0019 & $M_{\odot}$ \\
\hline
\end{tabular}




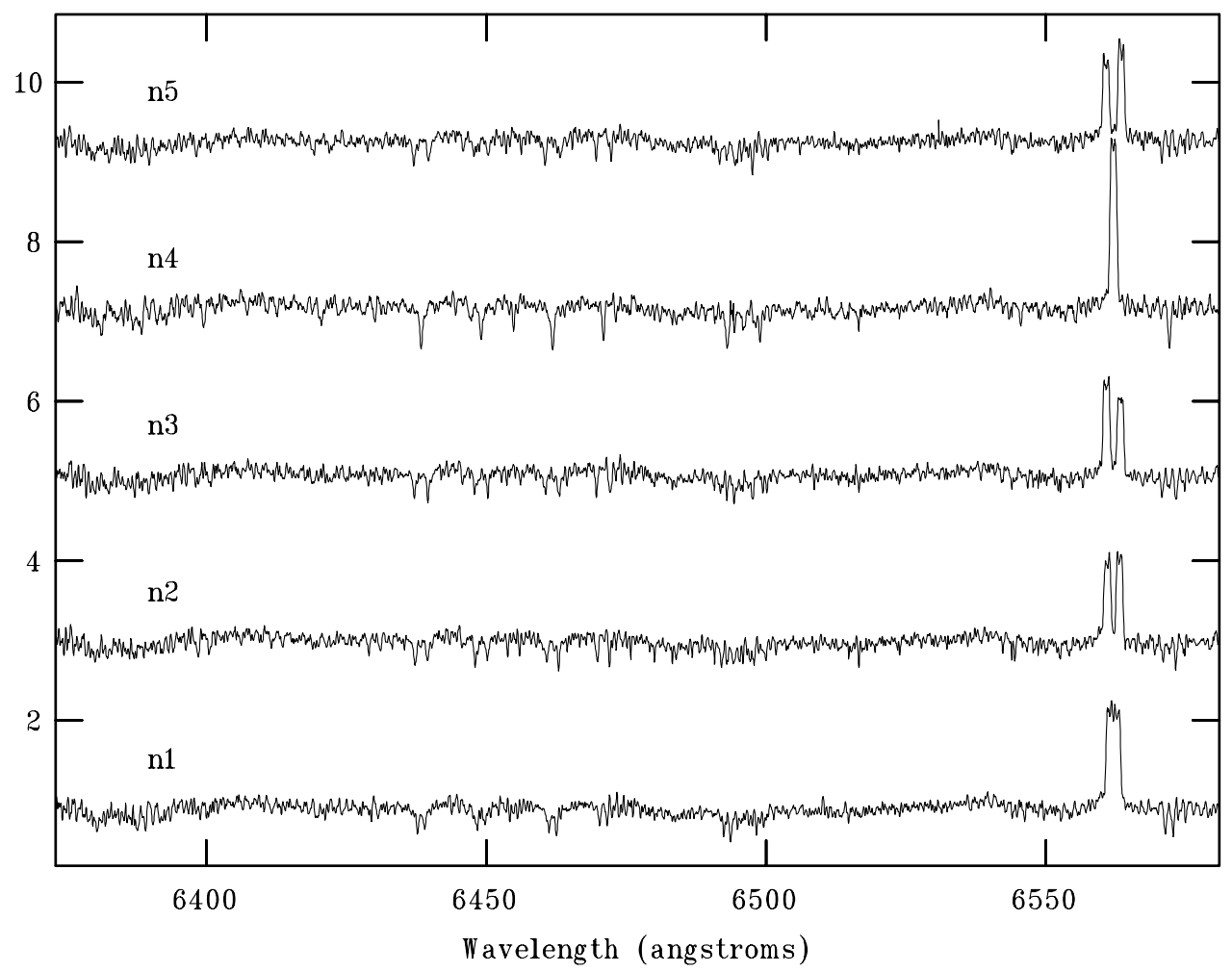

Figure 4. High-resolution spectra of EUVE J1501-43.6 in the $\mathrm{H} \alpha$ line region during our 5 nights of observations. Note the SB2 nature of the system in the photospheric absorption lines and in the chromospheric $\mathrm{H} \alpha$ emission line.

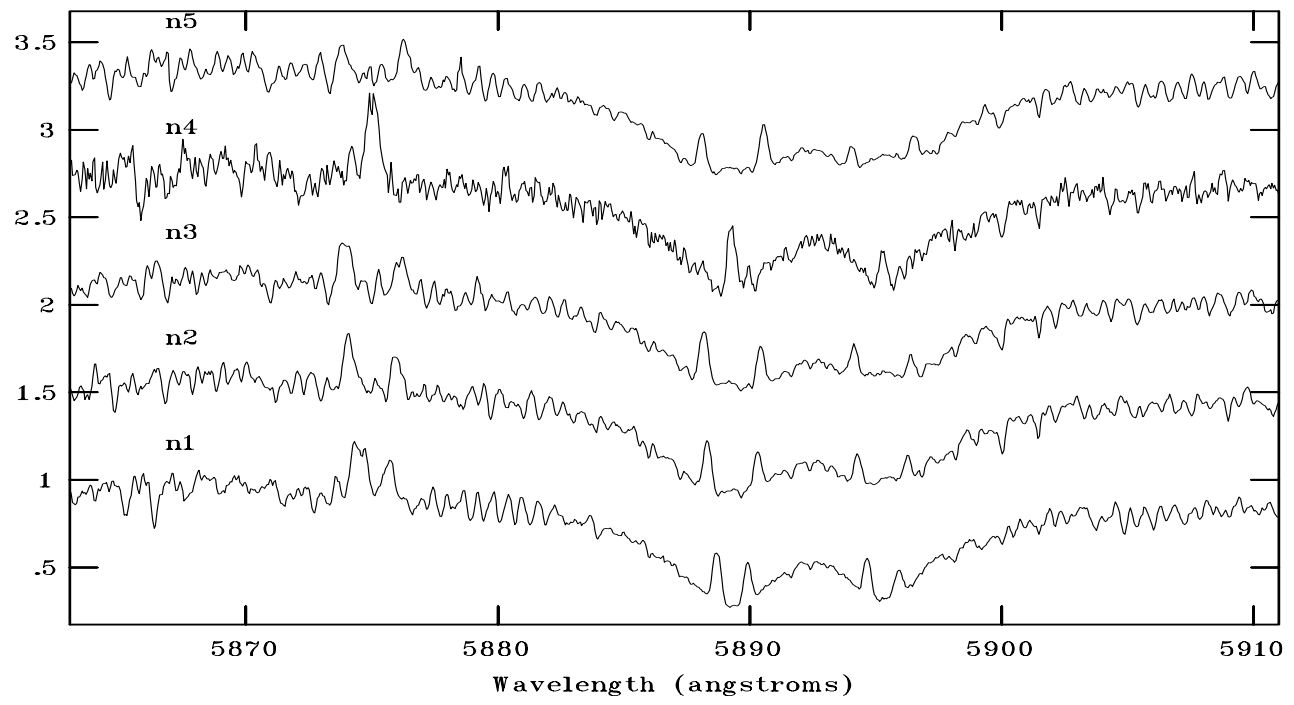

Figure 5. High-resolution spectra of EUVE J1501-43.6 in the region of the $\mathrm{Na}_{\mathrm{I}} \mathrm{D}_{1}, \mathrm{D}_{2}$, and the $\mathrm{He}$ I $\mathrm{D}_{3}$ lines. Note the clear emission from both components in these chromospheric lines. 

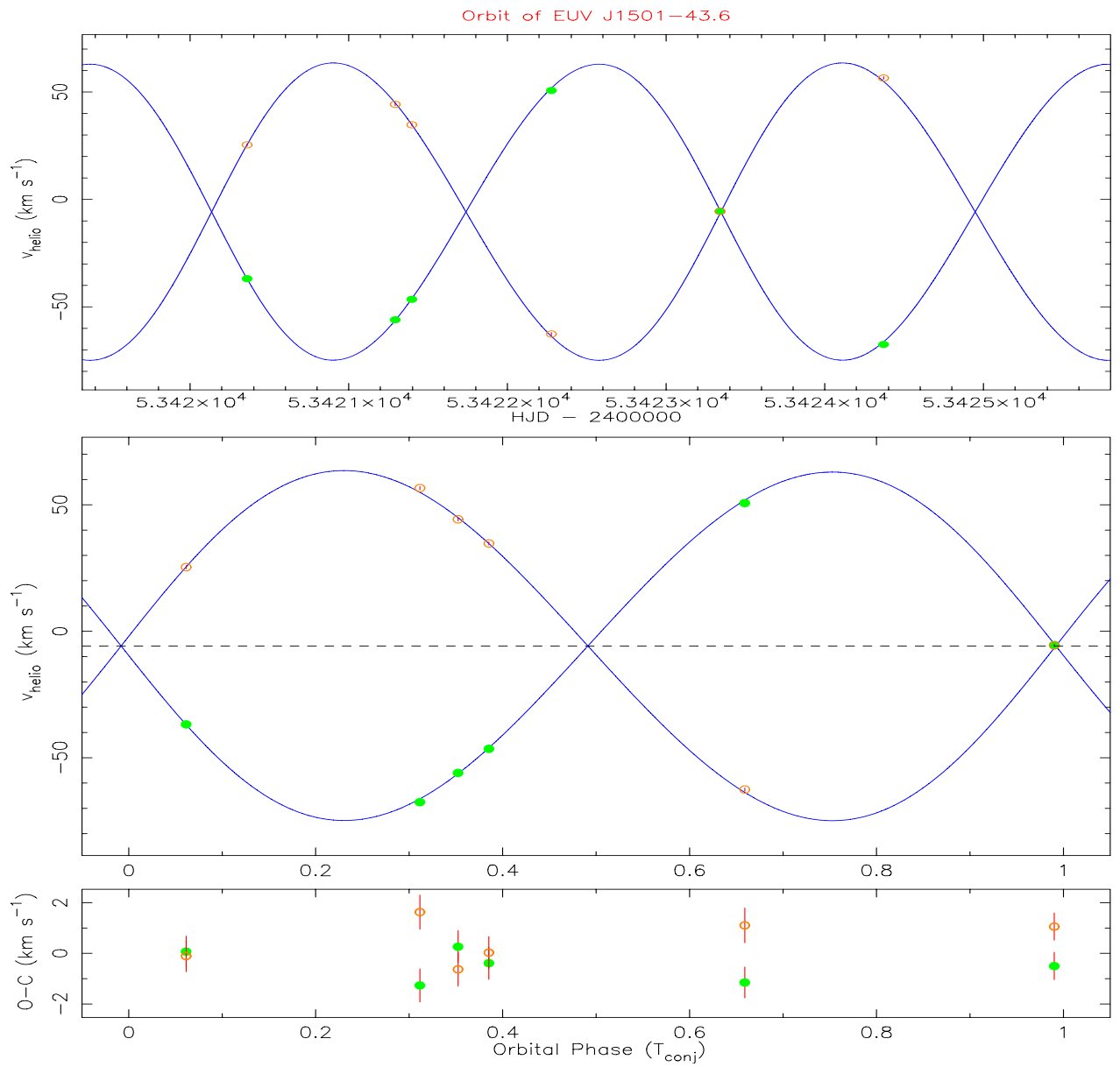

Figure 6. Heliocentric radial velocities determined by us vs. HJD (upper panel) and vs. orbital phase (lower panel). Our observations cover one and a half orbital period. Green solid circles represent the primary (the component with lower chromospheric emission) and red open circles the secondary. The blue solid line is the obtained orbital solution.

\section{Acknowledgements}

This work was supported by the Universidad Complutense de Madrid, the Spanish Ministerio de Educación y Ciencia (MEC), Programa Nacional de Astronomía y Astrofísica under grant AYA2005-02750, and the "Comunidad de Madrid" under PRICIT project S-0505/ESP-0237 (ASTROCAM).

\section{References}

Christian, D.J. \& Mathioudakis, M. 2002, AJ, 123, 2796

Craig, N., Christian, D.J., Dupuis, J., \& Roberts, B.A. 1997, AJ, 114, 244

ESA 1997, The Hipparcos and Tycho Catalogues, ESA SP-1200

Lampton, M., Lieu, R., Schmitt, J.H.M.M., Bowyer, S., Voges, W., Lewis, J., \& Wu, X. 1997, ApJS, 108, 545

Montes, D., Gálvez, M.C., Fernández-Figueroa, M.J., \& Crespo-Chacón, I. 2006, ApSS, 41, 